\title{
TESTING LINE OPTIMIZATION BASED ON MATHEMATICAL MODELING FROM THE METAMODELS OBTAINED FROM A SIMULATION.
}

\author{
Roberto L. Seijo-Vidal \\ University of Puerto Rico \\ College of Business Administration \\ Call Box 9000 \\ Mayagüez, PR 00681- 9000
}

\author{
Sonia M. Bartolomei-Suarez \\ University of Puerto Rico \\ Industrial Engineering Department \\ Call Box 9000 \\ Mayagüez, PR 00681- 9000
}

\begin{abstract}
This study is based on a real scenario in which simulation modeling is used in order to understand the behavior of the system. Sensitivity analysis, design of experiments, regression analysis for metamodeling purposes, and optimization are key elements of the simulation output analysis and are used in order to identify critical parameters and their relationship to multiple responses or output variables. Understanding this relationship allows to build mathematical expressions for the output variables which are the foundation for the optimization. Typical simulation optimization methods are not of practical value for this application. An optimization tool based on mathematical programming is developed. The tool is validated in terms of the metamodels' accuracy and the capacity to find a local optimum within the search region.
\end{abstract}

\section{INTRODUCTION}

This study is the continuation of a previous study in which focus was given to data gathering and analysis, and the development, verification, and validation of the simulation model. Different from the previous phase in which experimentation was done by means of a trial and error approach, this new phase focuses on the output analysis of the simulation model.

The system under consideration is a one-piece flow progressive assembly line. Each product is individually assembled, tested, final inspected, and packed. The testing area has been identified as the bottleneck and the area of major concern due to the high cost of the testing equipment, the high rejection rate and the excessive time to test a unit. Assembly capacity is available or relatively inexpensive to increase, contrary to the testing and final inspection areas. The study focuses on those two areas as well as packaging.

Figure 1 shows the conceptual model for the system under study. The testing line contains three areas: testing, final inspection and packaging. The products arrive from the assembly area to the testing area where they have to pass two different types of testing processes (Test \#1 and \#2) and a troubleshooting. Products that are accepted continue forward to the inspection area where they have to pass two different inspections (Inspection \#1 and Final Inspection) and another test (Test \#3). Products accepted continue forward to the packaging area. Products rejected at any stage are returned to the assembly area for repair. The handling of the products is done on a manual conveyor that takes advantage of gravity.

The objective of the study is to define the arrangement needed in terms of number of equipment and personnel in order to support the goal of increasing the throughput from a total of 800 units per month to 3000 units per month with minimum investment in terms of capital expenditures and operational costs. The above objective must be achieved considering the following restrictions or constraints: work-inprocess inventory must not exceed 22 units on average, equipment up-time (\%) and labor utilization (\%) 
must not exceed some pre-specified limits. This scenario is complicated by the fact that the rejection rate is high at the testing area and the testing process time is considered excessively high. Management has been working on improving these two issues but new performance levels are uncertain. For the purpose of the analysis, capital investment is related to the acquisition of new equipment and work-in-process inventory, and operational cost is mostly related to labor.

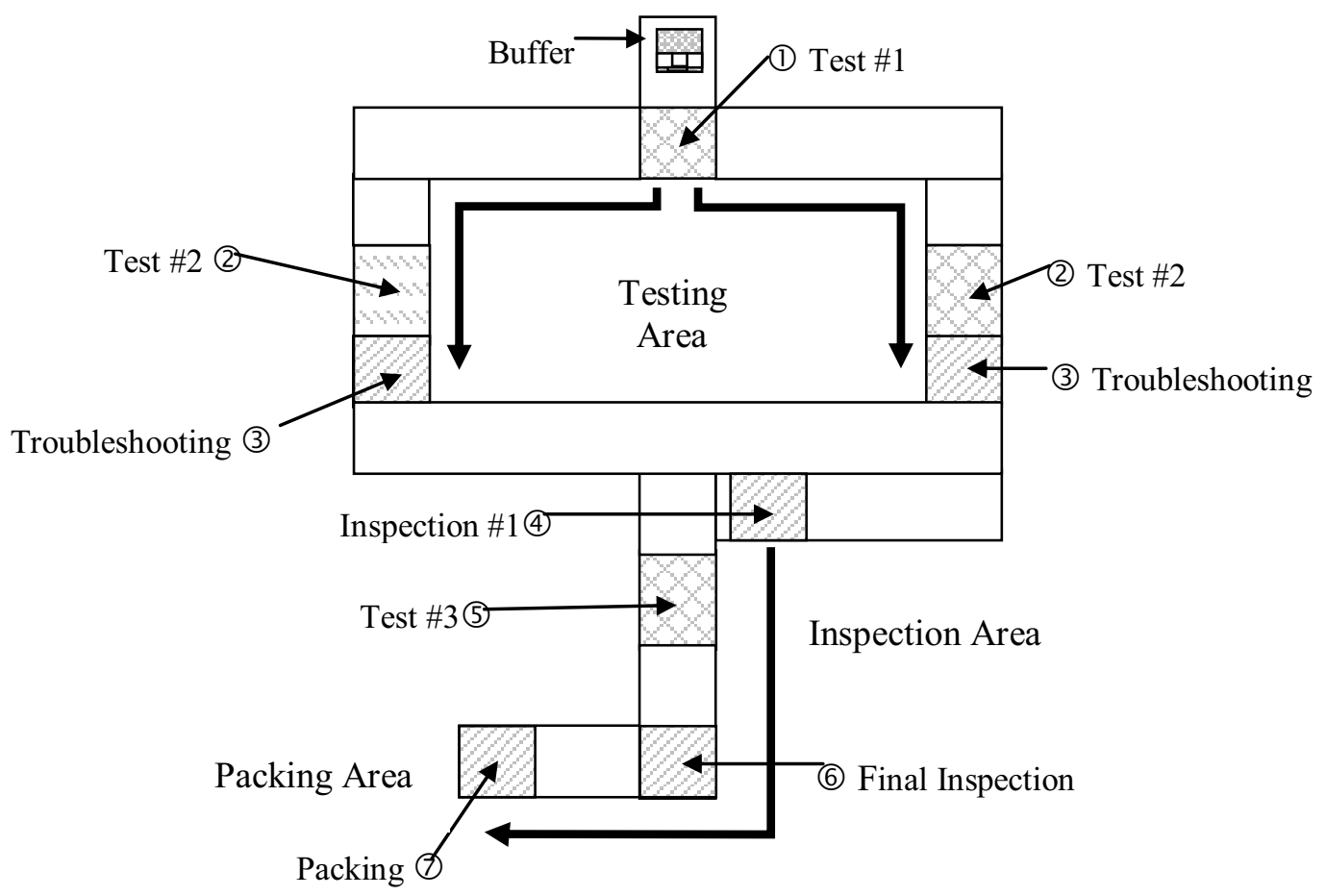

Figure 1. Conceptual model for the system under study

The analysis of simulation models of this complexity is usually simplified by reducing the search region, thus defining a manageable number of feasible scenarios. This is achieved by fixing or setting up input parameters to conditions that will positively impact the main goal of the analysis. For example, by fixing the yield to $90 \%$ instead of $70 \%$ and fixing the testing process time to $70 \%$ of the actual value instead of $85 \%$, we can expect more throughput and a reduction of the up-time for the testing machine which allows the expected volume to be reached with the least number of machines, thus reducing the capital expenditure cost. Nevertheless, the analysis has no value for the end user if those conditions for the input parameters are not reached in real life. The capacity for understanding the model is not only limited but there is no guarantee that the suggested alternative is the best in terms of reducing the capital and operational costs.

Understanding the relationship between the input parameters and multiple interrelated performance metrics or output variables with restrictions is key to achieving the expected goal of the system. Several input parameters are factors for which the end user can specify any value within a given range of interest. The fact that there are variable input parameters and multiple response variables with restrictions suggests the necessity for using computer simulation with design of experiments, metamodels, and optimization.

The expectation of this study is to provide the end user with an understanding of the behavior of the system within the given spectrum of the input parameters. Traditional simulation optimization methods are of no practical value for this problem due to the lack of resources availability from the end user and the uncertainty related to the values that the variable input parameters can assume. The approach followed is to express the relation between the simulation input parameters and the responses in terms of a mathe- 


\section{Seijo-Vidal and Bartolomei-Suarez}

matical optimization problem by use of metamodels. Even though the simulation literature recommends caution from the analyst side when pretending to solve simulation optimization problems with deterministic methods, mainly due to the inheriting variability of any simulation model, it is believed that this approach provides the end user with the full understanding of the system without having to invest in expensive simulation software. Microsoft's Excel Solver is used for the development of the optimization tool, showing to be of practical value due to its use of the Generalized Reduced Gradient (GRG) algorithm and its universality in today's business world.

\section{LITERATURE REVIEW}

As per Banks et al. (2001), output analysis is the examination of data generated by simulation and its purpose is to predict the performance of a system or to compare the performance of two or more alternative system designs. This definition suggests the importance of output analysis in any simulation study. Unfortunately, even though simulation is widely used for decision making in the industry, in many applications the analyst tends to follow the traditional trial and error approach instead of a scientific approach based on experimental design in order to understand the behavior of the system; therefore, undermining the power of simulation and increasing the probability for making erroneous inferences about the system.

The system being modeled is a non-terminal system and the interest is to analyze the model under the steady state condition. As per Sargent (2002), systems modeled for steady-state analysis introduce the complexities of: (1) removal of the bias of the imposed initial model state and (2) definition of a sample that admits an accepted estimate of sample variance, which is needed to determine the precision of estimates of steady-state parameters. The replications method is used in this study for the estimation of the sample variance.

As per Sánchez (2001), the purpose of preparing a simulation model is not to assess the capability of a single system, but to compare one or more systems to a standard level of performance, to compare several systems to one another, or to determine how the performance of one system changes according to particular variants of operating conditions. As per Kleijnen et al. (2001), sensitivity analysis is the systematic investigation of the reaction of the simulation responses to extreme values of the model's input or to drastic changes in the model's structure. Such an analysis helps identify the most important factors in a simulation study. Law et al. (1991) specifies that if the output is sensitive to some aspect of the model, then that aspect must be modeled carefully. For this study, sensitivity analysis is used for the purpose of identifying input parameters of the simulation model that should be considered as experimental factors as well as for defining the experimental levels for those factors. Hypothesis tests are used as part of the sensitivity analysis.

As per Montgomery (2001), experimental design is a test or series of tests in which purposeful changes are made to the input variables of a process or system so that we may observe and identify the reasons for changes that may be observed in the output response. In the context of simulation, the system or process is a computer model of the real system under study, either actual or planned. The simulation literature states that at the early stages of experimentation the analyst is concerned about which factors are important and how they might affect the responses. As the analyst understanding of the model's behavior progresses, the interest on experimentation turns from identifying critical factors to defining the optimal combinations of factor levels that maximize or minimize a response of interest. At this stage, the use of metamodels and response surface methodologies can be of great value. Once metamodels are constructed, the estimation of the gradient could also be of interest to the analyst for purposes of quantifying how the responses react to small changes in the quantitative factors as well as for optimization purposes. For our case, metamodels are developed for the experimental region with the purpose of optimizing a particular configuration of interest for the end user. Response surfaces are developed not for optimization purposes, but to confirm that an optimum for a particular configuration is within the experimental region.

Barton (1998) specifies that the major issues in metamodeling include: (1) the choice of a functional form for the regression model, (2) the experimental design and (3) the assessment of the adequacy of the metamodel. For the purpose of this study, regression models of first (linear) and second order (quadratic) 


\section{Seijo-Vidal and Bartolomei-Suarez}

are constructed from $2^{k}$ factorial and $2^{k-p}$ fractional factorial designs, and central composite designs, respectively. As for the metamodel adequacy, the test for lack of fit is done.

$\mathrm{Fu}(2001)$ defines simulation optimization as the optimization of performance measures based on outputs from stochastic (primarily discrete events) simulations. As in any optimization problem, the primary components are also present: input and output variables, objective function, and constraints. As per April et al. (2003), the main goal of simulation optimization is to find the combination of factor levels that minimizes or maximizes the objective function subject to the constraints imposed on factors and/or responses. The factors of interest are the ones that have the greatest effect on the responses as determined by the experimental designs. The challenge associated with finding the optimal combination of input parameters is that the relation (algebraic function) between the parameters and the response is unknown, which means that it has to be estimated. Swisher et al. (2000), states that the stochastic nature of the simulation output complicates the optimization problem. This may require multiple simulation runs (replicates) or long simulation runs to assure that the optimization algorithm is not misled by the variability of the response.

The simulation optimization techniques can be classified by the input parameters of the model since they are applied depending upon the type of input parameters, in particular, continuous or discrete, both being quantitative type of variables. In the case of qualitative or categorical variables, ranking and selection can be used. As per Swisher et al. (2000), in the case of continuous input parameters, methods may be classified as either gradient-based or non-gradient-based. When the method is applied on the entire (global) domain of interest, in principle, appropriate deterministic procedures can be applied to obtain an optimum. However, in practice, sequential response surface methodology is used rather than deterministic approaches when optimization is the main objective of the study (Kleijnen 1998, and Fu 2001).

Several techniques are available for optimization simulation when the input parameters are discrete. Ranking and selection and multiple comparison procedures can be applied when the set of alternatives is finite and small (Goldsman and Nelson 1998, and Boesel, Nelson, and Kim 2001). On the other hand, if the set of possible alternatives is infinite or large, ordinal optimization, simulated annealing, genetic algorithms, tabu search, and random search can be applied (Glover, Kelly, and Laguna 1999). All of the above methods for a large number of alternatives are known as meta-heuristic methods to simulation optimization, in which the simulation is run to define the function that relates the input parameters with the response variable, and then feedback into the meta-heuristic method to define a new set of alternatives and starting the process all over again.

For the system under consideration, the input variables of interest are both discrete (number of testing machines, personnel, and working shifts) and continuous (yield an testing time), adding to the complexity of the simulation output analysis and suggesting a strategic use of different optimization methods. This scenario is complicated by the fact that management needs a decision support tool not based on the interaction with simulation software. These observations are the main reason for the methodology that is explained in the following section.

\section{METHODOLOGY}

The objective of the study is to provide the end user with a tool that allows him/her to understand the behavior of the system for the whole spectrum of alternatives or possibilities as defined by the yield and testing process time. As previously mentioned, the scope is on the output analysis only since the validation of the simulation model was done during a previous phase not included in this study. It is important to emphasize that the scenario being studied is complex in nature based on the relationship between the input parameters and the multiple interrelated output variables, several restrictions or constraints imposed on the system, the uncertainty related to the yield and testing process time, and the fact that there are both continuous and discrete input parameters. Based on these observations, the methodology followed is summarized in this section. 


\subsection{Sensitivity Analysis}

Sensitivity analyses are done with respect to two main objectives: (1) to validate the warm-up period defined during the simulation analysis of the model for the actual (real) system, and (2) due to the high number of input parameters at the beginning of the study, to identify with the minimum simulation time effort the parameters that should be considered as experimental factors and their respective experimental levels. The methodology followed is practically the same for both cases. In general, the objective is to vary one factor at a time to determine its impact on the responses of the simulation. Any input parameter that is identified by means of hypothesis testing as statistically impacting at least one performance measure is considered as an experimental factor.

\subsection{Reducing the Problem with Experimental Designs}

The main objective at this stage of the simulation study is to reduce the number of critical factors to be considered in the metamodels. This is achieved through a series of sequential experimental designs with major focus on the categorical and discrete input parameters, in particular, the labor balancing issue, the number of assemblers, the number of final inspectors, and the number of draw out machines.

The expected result is to eliminate these factors from the critical list by fixing them to a level that would allow the model to achieve the objectives mentioned before. In order to do so, $2^{k-p}$ fractional factorial design with center points and $2^{k}$ factorial design are used. The sensitivity analyses are the base for setting the levels for each factor considered in the experiments.

\subsection{Development of Metamodels}

A series of parallel experimental designs are performed to develop the metamodels used in the optimization process of the study. These experimental designs focus on the testing area. Input parameters identified not as critical by the previous stages of the study are fixed to a particular set of values -thus- reducing the complexity of the model.

The testing area has four critical factors: inter-arrival time, number of testing machines, yield, and testing process time. All of the four critical factors are quantitative input parameters, but the number of testing machines is discrete. In addition, it is understood at this stage of the simulation study that some of the performance measures of interest could have a quadratic relationship with these factors. It is decided to run three parallel central composite experimental designs -one experiment for each number of possible testing machines $(3,4$, and 5$)$.

The evaluation of the outcomes from the experimental designs is based on statistical techniques generally used for these purposes, such as: hypothesis tests, analysis of variance, normality tests, run charts, surface plots, and a series of other commonly used graphical plots.

\subsection{Optimization Tool}

It would be easier for the analysis and execution of the study to fix the yield and testing process time to their respective maximum and minimum levels. Nevertheless, this would be of no practical value for the end user due to the uncertainty with respect to achieving those levels. Basically, the objective is to develop a tool that could be used for management decision making. This in fact is the complexity of the study since it forces us to understand the behavior of the system for the whole spectrum of alternatives or possibilities with respect to the yield and testing process time.

As previously mentioned, typical simulation optimization techniques are not practical options for the end user in terms of the availability of resources, contrary to developing a set of metamodels that could easily be used in exchange of the simulation model. The metamodels are used to build mathematical programming models for optimization purposes. These models are of the type of smooth non-linear optimization problems with 3 decision variables and 3 constraints. A smooth non-linear programming is one in which the objective function or at least one of the constraints is a continuous non-linear function of the 
decision variables. This characteristic suggests the use of the Generalized Reduced Gradient (GRG) algorithm, which is well known in the mathematical programming arena for solving optimization problems in which non-linear constraints and arbitrary bounds on the decision variables are allowed.

The standard Microsoft Excel Solver can solve smooth non-linear optimization problems using the GRG method for up to 200 decision variables and 100 constraints in addition to bounds on the variables. The code used by Microsoft Excel Solver is the GRG2, which is written in ANSI Fortran. It seeks a feasible solution first (if one is not provided) and then retains feasibility as the objective is improved. It uses a robust implementation of the Broyden-Fletcher-Goldfarb-Shanno (BFGS) quasi-Newton algorithm as its default choice for determining a search direction. A limited-memory conjugate gradient method is also available permitting solutions of problems with hundreds or thousands of variables. The problem's Jacobian is stored and manipulated as a dense matrix, so the effective size limit is one to two hundred active constraints (excluding simple bounds on the variables, which are handled implicitly). As per Boesel (2001), a special development of recent interest in simulation optimization is the integration of metaheuristic search with classical non-linear optimization such as the state-of-the-art GRG2.

All of the above suggests the use of MS Excel Solver and Visual Basic for Applications due to the easiness of use and the opportunity to develop an inexpensive optimization tool. Basically, the end user enters the values for the yield and testing process time, the tool selects the optimization model (for 3, 4 or 5 testing machines) and Microsoft Excel Solver finds the optimum based on maximizing an objective function and meeting the restrictions imposed by the metamodels. The search variable is the inter-arrival time. It is important to mention that the search is done within the feasible experimental region for each testing machine.

\section{METAMODELS AND VALIDATION OF OPTIMIZATION TOOL}

For each number of possible testing machines needed to achieve a throughput of 1000 units per shift (3000 units in three shifts), metamodels are built relating the input parameters (inter-arrival time, yield, and testing process time) to the output variables (throughput, up-time testing machine, and work-inprocess inventory). These metamodels become the constraints of the optimization problem. As per equation (1), the objective function is based on maximizing $Z$ which is defined as the monthly profit per shift, and is calculated as the revenue generated by the throughput less the cost related to work-in-process inventory and depreciation of the testing machines.

$$
Z=(S P)(T H P T)-(I C)(W I P)-\left(\frac{\left(\frac{\left(\frac{(E A C-B V)}{D Y}\right)}{12}\right)}{3}\right)(T M)
$$

Where $S P$ is the sales price per unit, $T H P T$ is the throughput, $I C$ is the inventory holding cost per unit per unit of time, WIP is the work-in-process, $E A C$ is the acquisition cost for a new testing machine, $B V$ is the book value at the end of the depreciation period, $D Y$ is the depreciation period in years, 12 is the number of months in a year, 3 is the number of shifts, and $T M$ is the number of new machines.

Note that $Z$ lacks the cost component associated with labor. This is mainly because the number of employees needed to achieve 1000 units per shift is fixed for the experimental region defined by the yield, the testing process time, and the inter-arrival time.

The validation of the optimization tool is done by focusing on two subjects: (1) predicting the accuracy of the metamodels vs. the simulation output, and (2) the ability of the experimental region to contain a 
local optimum with respect the combination of possible values for the yield and testing process time input parameters.

Table 1 Validation scenarios for the metamodels accuracy

\begin{tabular}{|c|c|c|c|}
\hline \multirow{2}{*}{ Scenario \# } & \multicolumn{2}{|c|}{ Input } & Output \\
\cline { 2 - 4 } & Yield (\%) & Testing Proc. Time (\%) & Optimization Model \\
\hline 1 & 90 & 70 & 3 Testing Machines \\
\hline 2 & 81.3 & 85 & 4 Testing Machines \\
\hline 3 & 72.6 & 100 & 5 Testing Machines \\
\hline
\end{tabular}

For subject (1), it is decided to run different scenarios for possible combinations of yield and testing process time (refer to Table 1). The output from the optimization tool is compared to the output of the simulation model. It is important to mention that for simulation purposes, the input parameter inter-arrival time is obtained from the optimization tool.

It is common to validate metamodels by means of confidence interval hypothesis tests. In this approach, the confidence interval for the prediction is statistically compared to the confidence interval of the simulation response. It is understood that for the case of this project, such a comparison is of no practical value for the end user or management of the company. The main reason being that management is more interested in knowing the probability distribution for the performance measure throughput, rather than the confidence interval for the mean (average throughput) at the stationary point. In other words, they were more interested in the behavior of the population at the stationary point. It is decided to do the validation by comparing the point estimate of the simulation for the responses of interest (throughput, up-time testing machines, utilization packing personnel, and work-in process inventory) vs. the prediction from the metamodels. The decision criteria for validation purposes are the same percentages used for estimating the number of simulation replicates at the time of doing the sensitivity analysis. These criteria are of practical value since they represent the allowable error for each performance measure given by the management of the company. It is understood that this validation approach is of common sense for management, thus gaining confidence on the results of the study.

In order to calculate the point estimate for the performance measures or simulation responses of interest, 1000 replicates are run for each validation scenario. Since 1 replicate is equivalent to a month worth of production, then 1000 replicates are equivalent to 83.3 years; from the point of view of the manufacturing life for this product, such amount of replicates are understood to be representative of the population. Statistically speaking, the confidence interval for each simulation response of interest is small enough to consider the simulation averages as the point estimates at the steady state. Table 2 presents the results for each validation run or scenario, and Table 3 presents the accuracy (error \%) of the metamodels vs. the simulation outputs or responses. Note that the error for each of the output variables of interest is less than the allowable error.

For subject (2), the optimization tool is run with two scenarios presented in Table 4. Scenario \#1 represents the limit for the 3 testing machines experimental region (in terms of yield and testing process time). Scenario \#2 is the limit for 4 testing machines. In the case of scenario \#1, the expectation is for the optimization tool not to find a feasible solution with the optimization model for 3 testing machines, but with the optimization model for 4 testing machines. This guarantees that for any combination of yield and testing process time feasible for 3 testing machines, the optimum is contained within the experimental region, at least within the limits provided for the yield and testing process time. The same being true for scenario \#2 and 4 testing machines, but in this case, the feasible solution is lying within 5 testing machines optimization model. This approach is valid because the combination of $90 \%$ yield, $70 \%$ of the testing process time, and $48.5 \%$ of the inter-arrival time, was a common point or the starting point for the de- 


\section{Seijo-Vidal and Bartolomei-Suarez}

velopment of the three experimental regions. In other words, the region for 3 testing machines is contained within the region for 4 testing machines, which in turn is contained within the region for 5 testing machines.

Table 2 Optimization tool and simulation results for validation purposes

\begin{tabular}{|c|c|c|c|c|c|c|c|c|c|c|c|c|}
\hline \multirow[b]{2}{*}{$\begin{array}{l}\text { \# } \\
\dot{0} \\
\text { లू } \\
\tilde{n}\end{array}$} & \multirow[b]{2}{*}{$\begin{array}{l}\overline{0} \\
\bar{\delta} \\
\dot{\Sigma} \\
\dot{0}\end{array}$} & \multicolumn{2}{|c|}{ Input } & \multirow{2}{*}{ 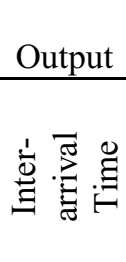 } & \multicolumn{4}{|c|}{ Simulation Model } & \multicolumn{4}{|c|}{ Optimization Tool } \\
\hline & & $\begin{array}{l}\frac{\partial}{2} \\
\frac{0}{0} \\
\vdots\end{array}$ & 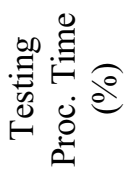 & & $\begin{array}{l}\text { हों } \\
\overrightarrow{0} \\
\vec{D} \\
\vec{E}\end{array}$ & 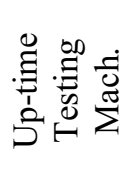 & $\frac{\beta}{3}$ & 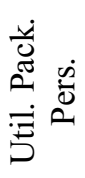 & $\begin{array}{l}\dot{\text { Dू }} \\
\overrightarrow{0} \\
\dot{E}\end{array}$ & 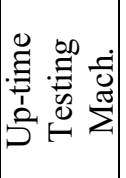 & $\stackrel{\beta}{3}$ & 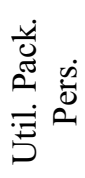 \\
\hline 1 & $3 \mathrm{TM}$ & 90.0 & 70.0 & 0.465 & 1096.2 & 94.5 & 19.7 & N/A & 1090.4 & 94.3 & 18.6 & N/A \\
\hline 2 & $4 \mathrm{TM}$ & 81.3 & 85.0 & 0.450 & 1021.2 & 86.7 & 13.7 & N/A & 1019.7 & 86.4 & 13.7 & N/A \\
\hline 3 & $5 \mathrm{TM}$ & 72.6 & 100.0 & 0.405 & 1016 & $\mathrm{~N} / \mathrm{A}$ & 15.5 & 77.6 & 1013.7 & N/A & 14.3 & 77.4 \\
\hline
\end{tabular}

Table 3 Accuracy (error \%) of the metamodel vs. the simulation

\begin{tabular}{|c|c|c|c|c|c|}
\hline \multirow{2}{*}{ Scen. \# } & Opt. Model & Through. & Up-time Testing Mach. & WIP & Util. Pack. Pers. \\
\cline { 3 - 6 } & & & & \multicolumn{3}{|c|}{$\%$ Error } \\
\hline 1 & $3 \mathrm{TM}$ & $0.53 \%$ & $0.21 \%$ & $5.58 \%$ & N/A \\
\hline 2 & $4 \mathrm{TM}$ & $0.15 \%$ & $0.35 \%$ & $0.00 \%$ & N/A \\
\hline 3 & $5 \mathrm{TM}$ & $0.23 \%$ & N/A & $7.74 \%$ & $0.26 \%$ \\
\hline \multicolumn{2}{|c|}{ Allowable Error } & $2.50 \%$ & $1.00 \%$ & $10.00 \%$ & $1.00 \%$ \\
\hline \multicolumn{2}{|r|}{} & & & & \\
\hline
\end{tabular}

Table 4 Validation Scenarios for the experimental regions

\begin{tabular}{|c|c|c|c|c|}
\hline \multirow{2}{*}{$\begin{array}{c}\text { Scenario } \\
\#\end{array}$} & \multicolumn{3}{|c|}{ Input } & \multicolumn{2}{|c|}{ Output } \\
\cline { 2 - 5 } & $\begin{array}{c}\text { Yield } \\
(\%)\end{array}$ & $\begin{array}{c}\text { Testing Proc. Time } \\
(\%)\end{array}$ & No Feasible Solution & Feasible Solution \\
\hline 1 & 85 & 75 & 3 Testing Machines & 4 Testing Machines \\
\hline 2 & 81.3 & 85 & 4 Testing Machines & 5 Testing machines \\
\hline
\end{tabular}

Table 5 shows that scenario \#1 has no feasible solution for 3 testing machines; instead, a feasible solution is obtained for 4 testing machines. Table 6 shows that scenario \#2 has no feasible solution for 4 testing machines; instead, a feasible solution is obtained for 5 testing machines. For validation purposes, these are the expected results.

The conclusion from tables 5 and 6 is that the experimental region (in terms of the yield and testing process time factors) for a particular number of testing machines, has the capability to contain the optimum for a particular combination of yield and testing process time if in reality there is a feasible solution within that particular number of testing machines. Validating the experimental region guarantees the right selection of the number of testing machines required for a particular combination of yield and testing 
time factors. It also shows the validity of the Generalized Reduced Gradient method as well as the usefulness of Microsoft Excel Solver for the optimization stage of this simulation study.

Table 5 Scenario \#1 - Experimental region

\begin{tabular}{|c|c|c|c|}
\hline \multirow{2}{*}{ Measure } & \multirow{2}{*}{ Constraints } & 3 Testing Machines & 4 Testing Machines \\
\cline { 3 - 4 } & $>=1000$ & 1000.0 & 1063.7 \\
\hline Throughput & $<=95 \%$ & 96.7 & 77.6 \\
\hline $\begin{array}{c}\text { Up-time Test. } \\
\text { Mach. }\end{array}$ & $<=22$ & 24.6 & 12.6 \\
\hline WIP & & \\
\hline
\end{tabular}

Table 6 Scenario \#2 - Experimental region

\begin{tabular}{|c|c|c|c|}
\hline \multirow{2}{*}{ Measure } & Constraints & Optimization Tool \\
\cline { 3 - 4 } & $>=1000$ & 971.4 & 5 Testing Machines \\
\hline Throughput & $<=95 \%$ & 96.6 & 1113.1 \\
\hline $\begin{array}{c}\text { Up-time Test. } \\
\text { Mach. }\end{array}$ & $<=22$ & 27.8 & N/A \\
\hline WIP & $<=85 \%$ & N/A & 16.1 \\
\hline $\begin{array}{c}\text { Util. Pack. } \\
\text { Pers. }\end{array}$ & & 85.0 \\
\hline
\end{tabular}

\section{CONCLUSIONS}

The objective of the study was to provide the end user with a tool that allows him/her to understand the behavior of the system for the whole spectrum of alternatives or possibilities as defined by the yield and testing process time. The scenario studied was complex in nature based on the relationship between the input parameters and the multiple interrelated output variables, several restrictions or constraints imposed on the system, the uncertainty related to the yield and testing process time, and the fact that there are both continuous and discrete input parameters.

The methodology followed proved to be crucial for the understanding of the system's behavior. It can be summarized as follows: 1) using sensitivity analysis and a series of sequential experiments with the objective of simplifying the complexity of the problem and identifying input parameters worthy of being considered for further experimentation and their respective experimental levels; 2) using experimental design for metamodeling purposes, and 3) developing an optimization tool with practical use for this application.

The optimization problem was of the smooth non-linear type requiring the use of the Generalized Reduced Gradient algorithm. The optimization tool was developed using Microsoft Excel Solver and Visual Basic for Application mainly because the standard Microsoft Excel Solver has the capability to implement the GRG by means of the GRG2 code, and the availability and practicability to the end user. 


\section{Seijo-Vidal and Bartolomei-Suarez}

It is important to mention that there was a trade-off between understanding the behavior of all possible combinations for the critical factors and the cost of developing such a tool. In the case of this study, it was relatively easy since the problem was already reduced to three quantitative critical factors (yield, testing process time, and inter-arrival time) for each number of testing machines. Note that as the number of factors increases, defining the experimental region becomes more difficult. In addition, the experimental region may tend not to fit a quadratic model, which complicates the analysis even further.

The complexity of this project was reduced by setting the throughput goal around 3000 units per month (1000 per shift). However, considering the throughput as uncertain adds on the complexity of the analysis since practically no input parameter could be set to a fix value. Solving such a system is worth trying from the academic point of view.

\section{REFERENCES}

April, J., F. Glover, J. P. Kelly, and M. Laguna. 2003. Practical introduction to simulation optimization. In Proceedings of the 2003 Winter Simulation Conference, ed. S. Chick, P. J. Sánchez, D. Ferrin, and D. J. Morrice, 71-78. Piscataway, New Jersey: Institute of Electrical and Electronics Engineers, Inc.

Barton, R. R. 1998. Simulation metamodels. In Proceedings of the 1998 Winter Simulation Conference, ed. D.J. Medeiros, E.F. Watson, J.S. Carson and M.S. Manivannan, 167-174. Piscataway, New Jersey: Institute of Electrical and Electronics Engineers, Inc.

Banks, J., J. S. Carson, B. L. Nelson, and D. M. Nicol. 2000. Discrete-event system simulation. 3rd ed. Upper Saddle River, New Jersey: Prentice-Hall, Inc.

Boesel, J., F. Glover, R. O. Bowden, J. P. Kelly, and E. Westwig. 2001. Future of simulation optimization. In Proceedings of the 2001 Winter Simulation Conference, ed. B. A. Peters, J. S. Smith, D. J. Medeiros, and M. W. Rohrer, 1466-1469. Piscataway, New Jersey: Institute of Electrical and Electronics Engineers, Inc.

Boesel, J., B. L. Nelson, and S. H. Kim. 2003. Using ranking and selection to "clean up" after simulation optimization. Operations Research 51(5):814-825.

Fu, M. C. 2001. Simulation optimization. In Proceedings of the 2001 Winter Simulation Conference, ed. B. A. Peters, J. S. Smith, D. J. Medeiros, and M. W. Rohrer, 53-61. Piscataway, New Jersey: Institute of Electrical and Electronics Engineers, Inc.

Glover, F., J. P. Kelly, and M. Laguna.1999. New advances for wedding optimization and simulation. In Proceedings of the 1999 Winter Simulation Conference, ed. P. A. Farrington, H. B. Nembhard, D. T. Sturrock, and G. W. Evans, 255-260. Piscataway, New Jersey: Institute of Electrical and Electronics Engineers, Inc.

Goldsman, D. and B.L. Nelson. 1998. Comparing systems via simulation. Chapter 8 in Handbook of Simulation: Principles, Methodology, Advances, Applications, and Practice, ed. J. Banks. New York: John Wiley \& Sons.

Kleijnen, J.P.C. 1998. Experimental design for sensitivity analysis, optimization, and validation of simulation models. Chapter 6 in Handbook of Simulation: Principles, Methodology, Advances, Applications, and Practice, ed. J. Banks. New York: John Wiley \& Sons.

Kleijnen, J. P. C., A. V. Noordegraaf, and M. Nielen. 2001. Sensitivity analysis of censored output through polynomial, logistic, and tobit regression meta-models: theory and case study. In Proceedings of the 2001 Winter Simulation Conference, ed. B. A. Peters, J. S. Smith, D. J. Medeiros, and M. W. Rohrer, 486-491. Piscataway, New Jersey: Institute of Electrical and Electronics Engineers, Inc.

Law, A. M., and W.D. Kelton. 1991. Simulation modeling and analysis. New York: McGraw- Hill, Inc.

Montgomery, D. C. 2001. Design and analysis of experiment. New York: John Wiley \& Sons, Inc.

Sánchez, S. 2001. ABC's of output analysis. In Proceedings of the 2001 Winter Simulation Conference, ed. B. A. Peters, J. S. Smith, D. J. Medeiros, and M. W. Rohrer, 30-38. Piscataway, New Jersey: Institute of Electrical and Electronics Engineers, Inc. 
Sargent, R. G., and R. E. Nance. 2002. Perspectives on the evolution of simulation. Operations Research 50(1):161-172.

Swisher, J. R., P. D. Hyden, S. H. Jacobson, and L. W. Schruben. 2000. A survey of simulation optimization techniques and procedures. In Proceedings of the 2000 Winter Simulation Conference, ed. J. A. Joines, R. R. Barton, K. Kang, and P. A. Fishwick, 119-128. Piscataway, New Jersey: Institute of Electrical and Electronics Engineers, Inc.

\section{AUTHOR BIOGRAPHIES}

ROBERTO L. SEIJO-VIDAL is an Assistant Professor in the College of Business Administration at the University of Puerto Rico, Mayagüez Campus (UPRM). He received a Ph.D. in Industrial Engineering from the University of Texas A\&M in August 2009. He teaches courses related to management operations and his research interests include the areas of supply chain, logistics and inventory, and simulation output analysis for complex systems. He has 15 years of industry experience working for multinational companies such as Westinghouse, Eaton-Cutler Hammer, and General Electric in several engineering and management positions. His email address is <roberto.seijo@upr.edu>.

SONIA M. BARTOLOMEI-SUAREZ is a Professor of Industrial Engineering at the University of Puerto Rico, Mayagüez Campus (UPRM). She received a PhD in Industrial Engineering from The Pennsylvania State University in 1996. Currently, she is the Co-PI for two projects: 1) College Access Challenge Grant sponsored by the Federal Education Department, and 2) UPRM ADVANCE Catalyst Program sponsored by the National Science Foundation. She is the professor in charge of the Industrial Engineering expertise on the Technology Transfer Program UPR-UPPR-TREN URBANO, a program that promotes the creation of multi-disciplinary teams of undergraduate students to perform applied research to design solutions to the public transportation problem in Puerto Rico. She has also acted as the Associate Dean of Academic Affairs of the College of Engineering. Her current research interests include: the integration of facilities planning and material handling systems, applying simulation and other industrial engineering tools to solve real life problems, and assess of the academic performance of the students of the College of Engineering. Her email address is <sonia.bartolomei eupr.edur. 